\title{
Characterization of community-based donation of personal protective equipment to an academic health center during the COVID-19 pandemic
}

\author{
Alexandra N. Fuher ${ }^{1}$, James T. Pathoulas ${ }^{1}$, Nathan Rubin ${ }^{2}$, Lisa M. Hursin ${ }^{3}$, Molly A. Wyman ${ }^{3}$, Ronda S. Farah*4 \\ ${ }^{1}$ University of Minnesota Medical School, Minneapolis, MN, United States \\ ${ }^{2}$ Biostatistics Core, Masonic Cancer Center, University of Minnesota, Minneapolis, MN, United States \\ ${ }^{3} \mathrm{M}$ Health Fairview, Minneapolis, MN, United States \\ ${ }^{4}$ Department of Dermatology, University of Minnesota, Minneapolis, MN, United States
}

Received: November 8, 2020

Accepted: December 10, 2020

Online Published: January 12, 2021

DOI: $10.5430 /$ jha.v9n6p33

URL: https://doi.org/10.5430/jha.v9n6p33

\begin{abstract}
Objective: The novel coronavirus 2019 (COVID-19) pandemic led to a shortage of personal protective equipment (PPE) early in the pandemic. Healthcare systems asked for public donations of PPE and established community drop-off sites. Herein, we aim to profile community PPE donors at one large academic medical center including evaluation of donor industry, public messaging, and psychosocial aspects of donation.

Methods: A survey was created and distributed to donors at two urban PPE drop-off sites between March and April 2020. Targeted donors and drop-off sites were located in the Twin Cities metropolitan area (approximate population of 3.5 million people).

Results: A total of 486 surveys were completed. Nearly half (47.3\%) of PPE donated was initially intended for personal use. Donors primarily learned of PPE collection efforts through word of mouth (23.2\%) and social media (22.7\%). The most frequently reported barrier to donation included distance between donors and drop off sites or location (27.8\%). Donors rated the severity of the PPE shortage in the state as a $7.8 \pm 1.7$ out of 10. There was a slight correlation between donors assessment of COVID-19 severity and feeling that their donation was a meaningful contribution against COVID-19 $(r=0.21, p=.00)$.

Conclusions: Future community collection campaigns during widespread disasters should prioritize mobilizing privately held goods from individuals rather than small businesses. Public messaging around donation should utilize simple narratives that are easily shareable via social media and evoke donation as a means of building community.
\end{abstract}

Key Words: Personal protective equipment, Coronavirus (COVID-19), Pandemic, Community, Donation

\section{INTRODUCTION}

The COVID-19 pandemic led to an acute increase in hospitalizations in the U.S. between March and May 2020, which caused increased demand for personal protective equipment (PPE) that exceeded supply. ${ }^{[1]}$ In response to PPE shortages, hospital systems simultaneously implemented policies to preserve existing PPE and sought new inventory. Strategies used to emergently direct resources to health systems during disasters typically rely on rerouting existing materials to sites with immediate needs. The shared impact of COVID-19

*Correspondence: Ronda S. Farah; Email: rfarah@umn.edu; Address: Department of Dermatology, University of Minnesota Health Cosmetic Lead; University of Minnesota, Minneapolis, MN, United States. 
on all health systems left these strategies untenable. In the absence of a national strategy for PPE centralization and distribution, health systems were left to individually secure PPE. Our academic health center (AHC) and physician practice plan established public PPE donation centers and engaged in public messaging efforts to route privately held PPE from the surrounding community to our hospital system.

Literature on the mobilization and distribution of aid to health systems in crisis is limited and primarily describes efforts directing existing regional aid to local disaster areas and AHCs serving as a central focus of the community response to disasters. ${ }^{[2,3]}$ No studies have evaluated community donation of vital supplies to health systems during a large-scale disaster, to our knowledge. The need to study community resource mobilization to healthcare systems is critical during the current COVID-19 pandemic, possible future pandemics, and future health disasters. Herein, we aim to profile community members who donated PPE to our AHC during the COVID-19 pandemic, including evaluation of donor industry, public messaging, and psychosocial aspects of donation.

\section{MethodS}

This study was deemed non-human research by the University of Minnesota Institutional Review Board (STUDY 00009368). A 29-question survey was created and distributed to donors at two urban PPE drop-off sites between March and April 2020. Targeted donors and drop-off sites were located in the Twin Cities metropolitan area (approximate population of 3.5 million people). The survey was hosted on a secure web-based platform, Qualtrics. Donors accessed the survey while inside their vehicles via $Q R$ codes posted at donation drop-off sites and they completed the survey onsite. The survey asked respondents to characterize items for donation, method by which they heard of donation, opinion on severity of COVID-19 in the state, feelings of community and contribution at time of donation, and barriers to donation. Incomplete responses were omitted.

\section{Statistical methods}

Mean scores and standard deviations for survey responses by intended use were completed. Simple linear regression was used to analyze the relationship between survey responses and date. Pearson correlation test was used to compare respondents' feelings towards donation. An alpha of 0.05 was used to determine statistical significance. $\mathrm{R}$ version 3.6.0 was used for the analysis.

\section{Results}

\subsection{Survey response \& donor characteristics}

A total of 486 surveys were completed over 22 days. Survey responses gradually declined over time, with a large dip at the end of week 1 (see Figures 1 and 2). Nearly half (47.3\%) of PPE donated was initially intended for personal use (see Table 1). Donors learned of PPE collection efforts through word of mouth (23.2\%), social media $(22.7 \%)$, and telephone calls $(1.6 \%)$ (see Table 2 ). The proportion of donors learning of collection efforts by word of mouth remained stable over time (see Figure 1). Donors who reportedly learned of collection efforts through social media increased from week 1 to week 2 of the PPE collection drive.

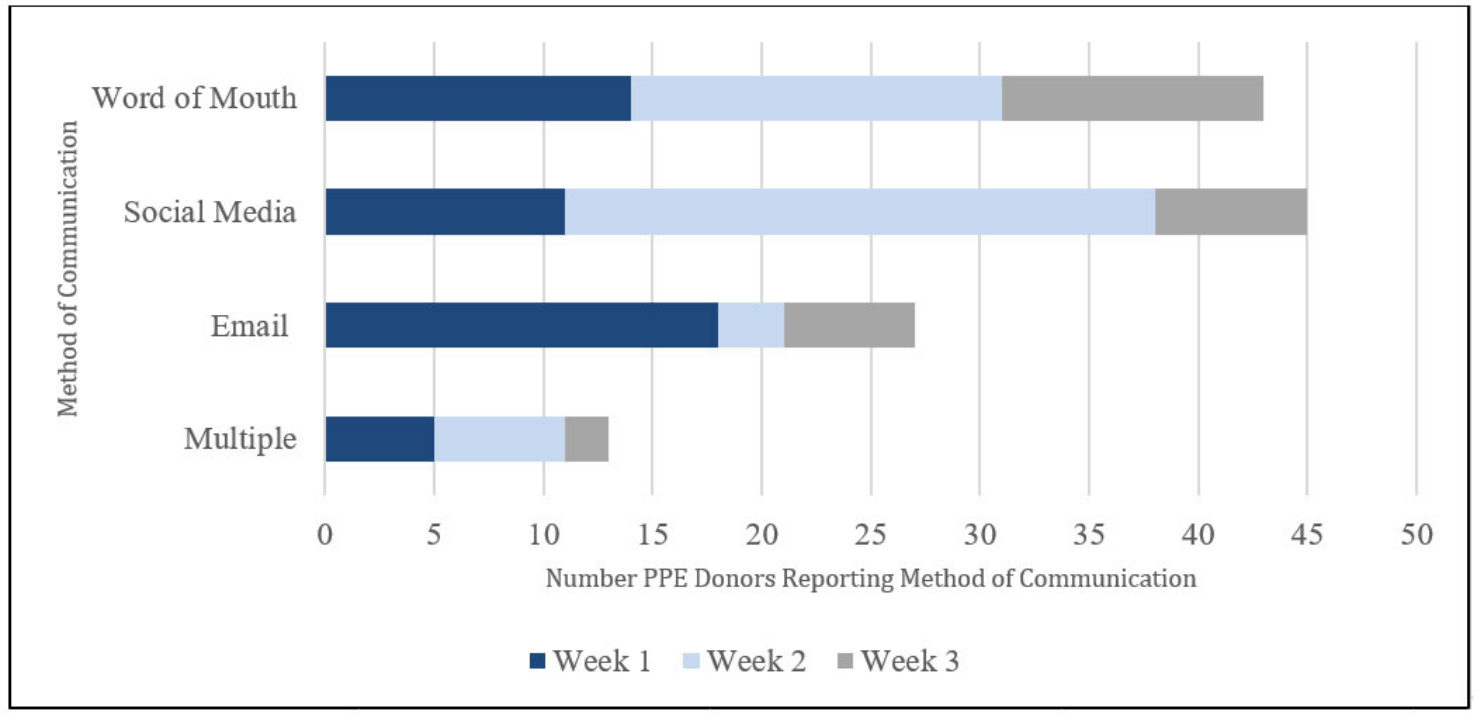

Figure 1. Method by which PPE donors heard of PPE collection efforts by week 


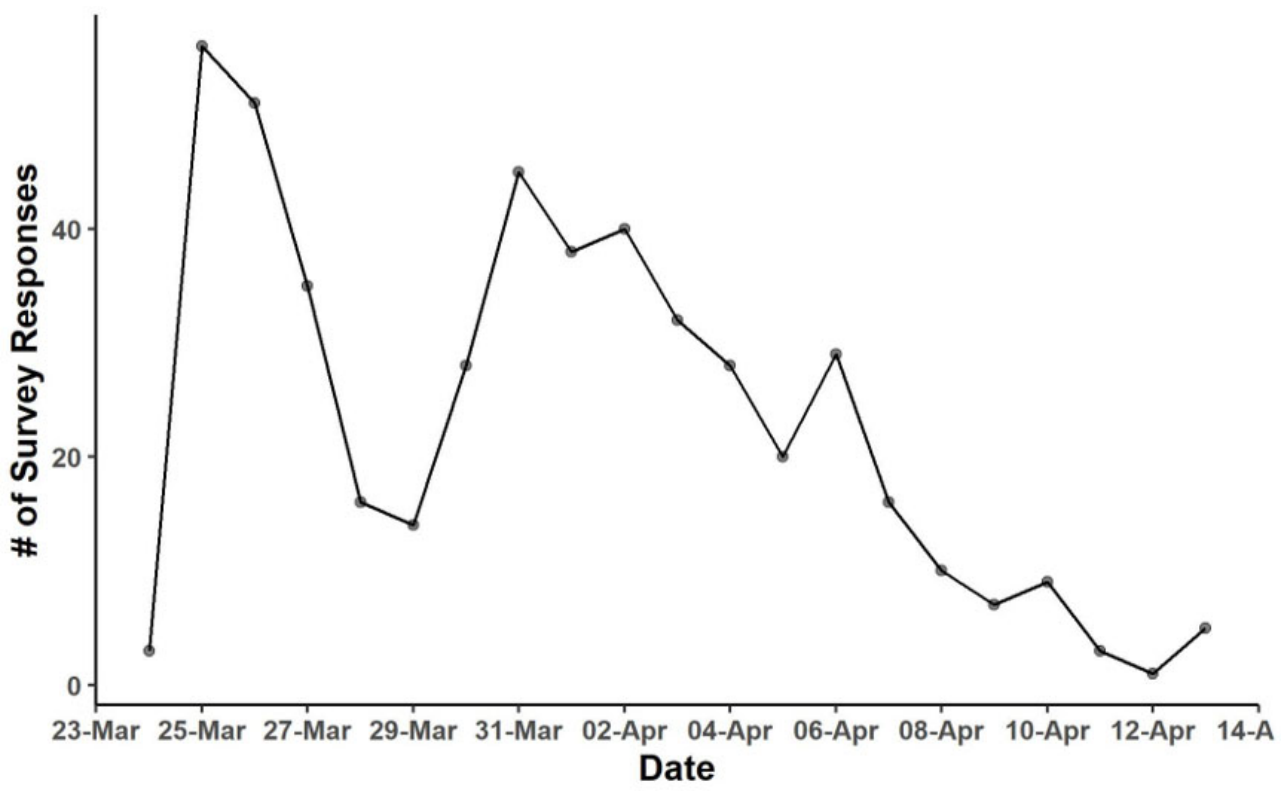

Figure 2. Frequency of survey responses over time

Survey response are a proxy for number of unique PPE donations. A gradual decline in donations over time is observed. Donations peaked on March 25, 2020, the same day the Governor instituted an executive stay-at-home order. ${ }^{[5]}$ Donations decreased sharply between March 27 and March 31, 2020, the same time a PPE social media campaign was launched.

Table 1. Intended use of PPE donated by community to an academic health center during COVID-19

\begin{tabular}{ll}
\hline Category & $\begin{array}{l}\text { Proportion of Total Donations } \\
(\mathbf{N}=\mathbf{1 4 8})\end{array}$ \\
\hline Personal Use & $70(47.3 \%)$ \\
Business & $24(16.2 \%)$ \\
Independent or Private Clinic & $4(3.4 \%)$ \\
Research & $3(2.0 \%)$ \\
Educational & $1(0.7 \%)$ \\
\hline
\end{tabular}

\subsection{Barriers to donation}

The reported barriers to donation included distance between donors and drop off sites or location (27.8\%), as well as logistics involved in the drop-off process (22.2\%) (see Table $3)$.
Table 2. Method of communication by which donors heard of PPE collection efforts

\begin{tabular}{ll}
\hline Method & $\begin{array}{l}\text { Proportion of PPE Donors } \\
(\mathbf{N}=\mathbf{1 8 5})\end{array}$ \\
\hline Word of Mouth (family, friend, etc.) & $43(23.2 \%)$ \\
Social Media & $42(22.7 \%)$ \\
Email & $27(14.6 \%)$ \\
Online, unspecified & $22(11.9 \%)$ \\
News & $15(8.1 \%)$ \\
Multiple & $13(7.0 \%)$ \\
Other & $11(5.9 \%)$ \\
Organization employee & $8(4.3 \%)$ \\
Phone Call & $3(1.6 \%)$ \\
Unsure & $1(0.5 \%)$ \\
\hline
\end{tabular}

Table 3. Barriers to donation reported by PPE donors

\begin{tabular}{ll}
\hline Method & Responses $\mathbf{( N = 3 6 )}$ \\
\hline Drop-off distance/location & $10(27.8 \%)$ \\
Drop-off Process/logistics & $8(22.2 \%)$ \\
Homemade mask material shortage & $4(11.1 \%)$ \\
Lack of mail-in instructions & $2(5.6 \%)$ \\
Uncertain about safety or acceptability of homemade mask donations & $1(2.8 \%)$ \\
Uncertain point of contact & $1(2.8 \%)$ \\
None & $1(2.8 \%)$ \\
Other, unspecified & $9(25.0 \%)$ \\
\hline
\end{tabular}




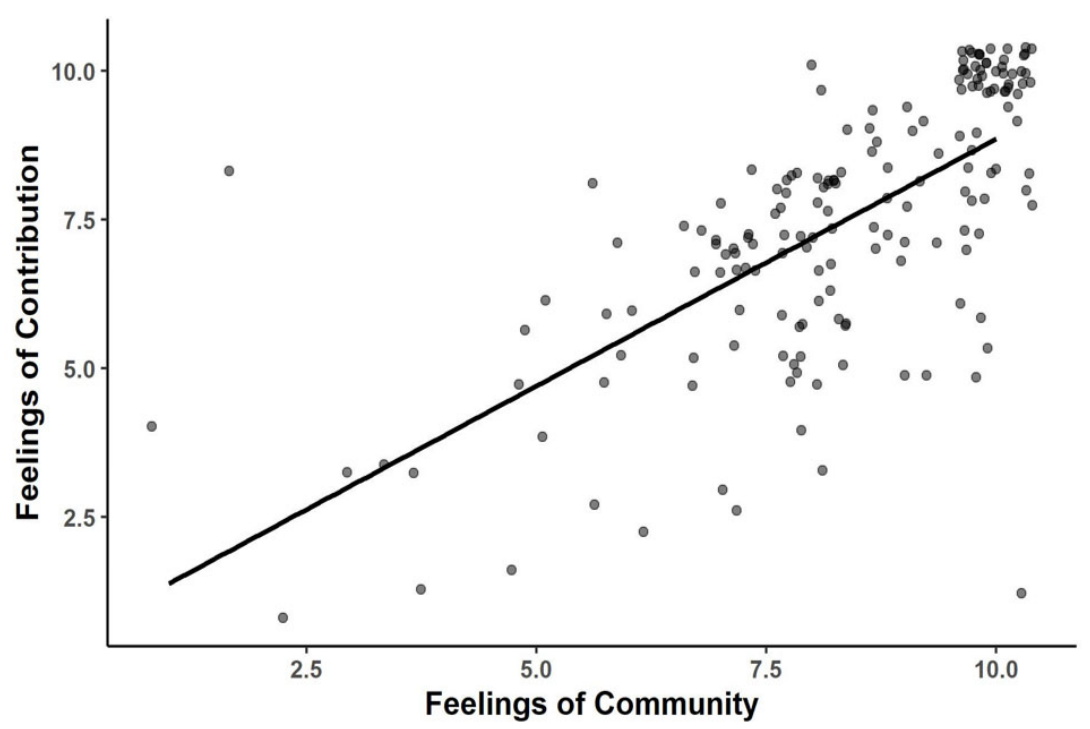

Figure 3. Psychosocial aspects of PPE donation

PPE donors for whom the act of donation engendered a sense of shared community also felt that their PPE donation made a difference in community-wide efforts to combat COVID-19.

\subsection{Psychosocial aspects of donation}

Psychosocial aspects of donation were defined as the degree to which donors felt their act of donation engendered a sense of shared community and the degree to which donors felt their PPE donation contributed to overall community efforts against COVID-19. Donors rated the severity of the PPE shortage in our state as a $7.8 \pm 1.7$ out of 10 . Donors also reported that having the ability to donate PPE led to a strong degree of community and contribution (see Figure 3 ).

PPE donors who ranked the severity of the COVID-19 pandemic as more severe did not have a higher sense of contributing to combat COVID-19 as a result of having the ability to donate $(r=0.08, p=.28)$. Donors who ranked the severity of the COVID-19 pandemic as more severe tended to feel a greater sense of shared community through PPE contribution, but the correlation was only slight $(r=0.21, p=.00)$. There was a strong positive statistically significant correlation between donors' sense of shared community through donation and donors' feelings that their actions were contributing to pandemic efforts $(r=0.68, p=.00)$ (see Figure 3).

\section{Discussion}

AHC leadership had to evaluate several unique considerations to establish a PPE donation program during the COVID19 pandemic. Social distancing directives, including a statemandated shelter in place order, posed a challenge to donors reaching in-person donation sites. The COVID-19 pandemic also posed a challenge to public messaging about PPE donation, as distancing directives had the potential to reduce in-person interactions and limit the effectives physical public advertisements including print news, banners, and billboards. However, regional news widely covered a nurses' PPE march on the Capitol and an Executive Order in the state that required businesses to report their inventory of privately held PPE to the state, which raised public awareness of the PPE shortage. $^{[3,4]}$

The acute PPE shortage experienced by our AHC was urgent and there was a lack of peer-reviewed literature that could be used to inform an evidence-based approach to PPE collection. Without time to introduce a proof-of-concept plan for a PPE collection effort that could later be scaled, collection efforts relied on key assumptions. First, that private individuals, organizations, and businesses in our region had a sufficient volume of PPE and had the desire to donate to our AHC. Second, that decreased in-person interactions during the COVID-19 pandemic would render word of mouth an ineffective form of public messaging and efforts to raise awareness of PPE collection efforts should instead be focused on phone calls and social media outreach. While the surrounding community showed remarkable support through donating a high volume of PPE to our AHC early in the pandemic, our experience made apparent the need for studies examining donation of community resources to health systems during periods of widespread disaster.

\subsection{Donor characteristics}

Our initial campaign efforts targeted businesses and private clinics, with phone calls being the predominant mode of communication used in the initial PPE collection campaign. Unexpectedly, business and clinics represented less than $20 \%$ 
of donations, while approximately half of donated PPE was intended for personal use (see Table 1). Furthermore, phone calls were the least reported mode of communication by which donors learned of PPE collection efforts. Alternatively, word of mouth was reportedly the leading mode of communication with donors, despite pandemic-related social isolation. Future collection efforts, therefore, should target individuals with a clear initial message that can be distributed easily by word of mouth or social media.

Treating survey responses as a proxy for donors, the number of individual donations trended down over the survey collection period in a bi-modal distribution. Of note, donations started trending up again the day following the start of the AHC's social-media campaign to raise awareness for PPE donation drop off sites (see Figure 2).

\subsection{Barriers to donation}

The major barrier to donation involved drop-off distance, location, and logistics. For the PPE donation campaign being surveyed, two drop-off locations were established, and the drop-off process was not publicized in initial collection efforts. Future collection efforts should offer several dropoff locations and publicize the drop-off process for donors. Additionally, to address other reported barriers to donation the process for mail-in donations should also be made public, and a point contact person should be established, if feasible.

\subsection{Psychosocial aspects of donation}

Donors who felt their PPE donation made a meaningful contribution to frontline healthcare workers also felt that the act of PPE donation engendered feelings of shared community, regardless of how they felt about the severity of the pandemic (see Figure 3). Future public messaging regarding community donation should leverage this connection, highlighting donation as a method of strengthening shared community instead of centering on disaster severity, as we found it did not increase donors' sense of contribution. These community contribution narratives are especially important if donors' sense of contribution positively correlates with the individual decision to donate, although this remains unknown.

\subsection{Limitations}

We recognize there are several limitations to our study. For one, the survey was distributed to donors; the perspective of non-donors was excluded and needs to be further evaluated. The survey-based nature of that was distributed via convenience sampling methods limits the external validity of our results.

\section{Conclusion}

Examining PPE donation efforts during the COVID-19 pandemic is needed to inform future strategies of mobilizing community resources during pandemics or other resourcelimited situations. Our study evaluated three key aspects of donation efforts. First, future collection campaigns should target community individuals more so than small businesses and private practices. These campaigns should craft messages that can be easily distributed via word-of-mouth and social media. Second, the campaigns should maximize dropoff locations and clearly publicize the procedure for donation drop-off. Third, messages of collection efforts should focus on narratives that evoke donation as a means of building community.

\section{ACKNOWLedgements}

We acknowledge the contributions of the Academic Health Center team who coordinated PPE drop-off sites and survey collection. We would also like to acknowledge the more than fifty University of Minnesota medical students who volunteered to assist in contacting potential donors.

\section{FUNDING}

Research reported in this publication was supported by NIH grant P30 CA77598 utilizing the Biostatistics and Bioinformatics Core shared resource of the Masonic Cancer Center, University of Minnesota and by the National Center for Advancing Translational Sciences of the National Institutes of Health Award Number UL1-TR002494. The content is solely the responsibility of the authors and does not necessarily represent the official views of the National Institutes of Health.

\section{CONFlicts OF InTEREST Disclosure}

The authors declare they have no conflicts of interest.

\section{REFERENCES}

[1] Lamberts B. Nurses plan march on Minnesota Capitol to protest lack of PPE. Minn Post. May 11, 2020 [Accessed May 25, 2020]. Available from: https: //www.minnpost . com/glean/2020/05/nurses-plan-m arch-on-minnesota-capitol-to-protest-lack-of-ppe/

Published by Sciedu Press
[2] Charney RL, Rebmann T, Dalawari P, et al. Public Expectations of Hospitals to Provide Resources and Services to the Uninjured During Disasters: A Qualitative Study. Health Security. 2016; 14(6): 389-396. PMid: 27835039. https : //doi.org/10.1089/hs. 201 6.0059

[3] Dunlop AL, Logue KM, Beltran G, et al. Role of Academic In- 
stitutions in Community Disaster Response Since September 11, 2001. Disaster Med Public Health Prep. 2011; 5(3): 218-226. PMid: 22003139. https://doi.org/10.1001/dmp.2011.75

[4] Melo F. M Health Fairview accepting handmade masks, wipes, sanitizer and more at University Avenue drop-off site. Pioneer Press; March 30, 2020. Available from: https: //www.twincities.com/2020/03/30/m-health-fairvie w-accepting-handmade-masks-wipes-sanitizer-and-mor e-at-university-avenue-drop-off-sitecoronavirus/

[5] State of Minnesota Executive Department. Governor Tim Walz. Emergency Executive Order 20-20. Directing Minnesotans to Stay at Home. March 30, 2012 [Accessed October 11, 2020]. Available from: https: //mn.gov/governor/assets $/ 3$ a. $\% 20 \mathrm{EO} \% 202020$ $\% 20$ FINAL $\% 20$ SIGNED\%20Filed_tcm1055-425020.pdf 